\title{
HACIENDO-LUGAR VÍA HUELLAS Y APEGOS: LAS PERSONAS MIGRANTES AFRICANAS Y SUS EXPERIENCIAS DE MOVILIDAD, INMOVILIDAD E INSERCIÓN LOCAL EN AMÉRICA LATINA INTRODUCCIÓN AL DOSSIER TEMÁTICO REMHU 56
}

\section{Place-making via traces and attachments: African migrants and their experiences of mobility, immobility and local insertion in Latin America. Introduction to the thematic dossier REMHU 56}

Nanneke Winters*

Franziska Reiffen ${ }^{* *}$

\begin{abstract}
Resumen. Este artículo introductorio al dossier temático traza los conocimientos y estudios existentes sobre la actual migración africana subsahariana hacia y por América Latina, y propone una perspectiva del haciendo-lugar para su análisis. Tal enfoque analítico permite incluir y vincular las experiencias y prácticas de las personas migrantes con una mirada más estructural que tiene en cuenta regímenes de movilidad y la industria de la migración. Más allá de una victimización o esencialización de las personas migrantes africanas, así se puede llegar a una mejor comprensión de sus compromisos de corta o larga duración con los lugares en los cuales habitan o que atraviesan en sus trayectorias; compromisos que afectan y se desarrollan tanto a un nivel material como social y emocional.
\end{abstract}

Palabras clave: trayectorias de migración africana; inmovilidad; haciendolugar; regímenes de movilidad; la industria de la migración.

\begin{abstract}
The introduction to this thematic dossier traces existing scholarship on current sub-Saharan African migration to and through Latin America. For analysing this migration, we propose a place-making perspective. Such a perspective enables us to illuminate the articulations between migrants' everyday experiences and structural conditions of mobility regimes and the migration industry. Going beyond victimizing or essentializing African migrants, this approach allows a better understanding
\end{abstract}

Instituto de Antropología y de Estudios Africanos de la Universidad Johannes Gutenberg de Mainz, Alemania. Mainz, Alemania. E-mail: nanneke.winters@uni-mainz.de. Orcid: 00000002-8036-3690.

* Instituto de Antropología y de Estudios Africanos de la Universidad Johannes Gutenberg de Mainz, Alemania. Mainz, Alemania. E-mail: reiffen@uni-mainz.de. Orcid: 0000-0001-9118-4308. 
of migrants' long or short-term engagements with the places in which they live or which they cross during their trajectories, an engagement that entails material, social and emotional aspects.

Keywords: trajectories of African migration; immobility; place-making; mobility regimes; migration industry.

\section{Introducción ${ }^{1}$}

En este dossier temático, pretendemos abordar dinámicas y experiencias de la actual migración africana subsahariana hacia y por América Latina. Según la Organización de los Estados Americanos y la Organización Internacional para las Migraciones (GS/OAS, IOM, 2016), en las últimas dos décadas el continente no sólo ha visto más migración sino también más diversidad migratoria, incluyendo la presencia de personas migrantes desde países caribeños, asiáticos y africanos. En general, esta situación está vinculada con las cada vez mayores restricciones migratorias en Europa y los Estados Unidos, por un lado, y las políticas de migración más abiertas en América Latina por otro. Al mismo tiempo, como destaca Soledad Álvarez Velasco:

si bien la región Centro y Sudamericana históricamente respondía desde una lógica de aperturismo y un enfoque de Derechos Humanos frente a la movilidad humana, actualmente parece que en la región se ha puesto en marcha un régimen de control y securitización frente a la migración; análogo a regímenes globales. (Álvarez Velasco, 2016, p. 31)

Sin embargo, el carácter complejo de estos flujos dificulta las políticas de detección, detención y deportación de personas migrantes. Además, América Latina es tanto región de llegada y de destino como de tránsito. En este dossier, no intentamos alisar esta complejidad, sino más bien utilizarla para, en primer lugar, reconocer los vínculos entre la migración sur-sur y sur-norte, la forzada y la voluntaria, y, en segundo lugar, poner énfasis en la volatilidad de la migración, su fluidez y sus huellas, para así contribuir a una mejor comprensión del tema.

Basándonos en conceptos tanto de la antropología y la geografía como de los estudios de migración, en esta contribución buscamos explorar el haciendo-lugar de las personas migrantes africanas en las localidades que habitan o por las cuales pasan en sus trayectorias por América Latina.

\footnotetext{
1 Este dossier temático surgió a base del simposio "Experiencias actuales de migrantes y refugiados africanos en América Latina", que tomó lugar durante el 56으 Congreso Internacional de Americanistas (ICA) en la Universidad de Salamanca, España, en julio 2018. Nanneke agradece al Institute of Development Policy (IOB) en Amberes por su apoyo institucional y a la Research Foundation - Flanders (FWO) por la beca que hizo posible asistir a este simposio. Las dos agradecemos a las participantes por su valiosa contribución al simposio y su dedicación a los artículos en el dossier. También nos gustaría agradecer al editor y los y las revisores de REMHU, por brindarnos la oportunidad de armar y finalizar este dossier.
} 
Tomando en cuenta una combinación compleja de los regímenes e industrias de la migración, la volatilidad de las trayectorias de las personas migrantes, y la combinación entre hospitalidad y hostilidad que encuentran en las localidades de su recepción, intentemos ofrecer una mejor comprensión de las prácticas de haciendo-lugar. A pesar de las posibles limitaciones de su estancia legal, a pesar de sus posibles imaginaciones y estrategias de movilidad, y a pesar de las posibles dificultades que encuentran para establecerse en América Latina, estas personas migrantes se insertan localmente y apropian espacios locales, dándoles sentido. Para explorar tales prácticas, nos basamos en el trabajo de la antropóloga Annika Lems, quien plantea la pregunta de cómo nosotros/-as como investigadores podemos vincular los compromisos diarios de las personas migrantes con un cierto lugar con un análisis de las estructuras de poder que influyen sobre estos compromisos (Lems, 2018, p. 34), como el control migratorio, el mercado laboral, y la xenofobia. Las experiencias y prácticas de las personas migrantes, quienes luchan para dar un sentido a su estancia en cierta localidad, apropiándola, transformándola en un lugar social, afectan y están afectadas por las configuraciones sociales y materiales de las localidades. Siguiendo al término que utiliza el antropólogo Arturo Escobar (2010), a tales experiencias y prácticas de las personas migrantes se puede referir como un proceso de "haciendo-lugar".

En este dossier temático, ponemos en conversación autores/-as quienes estudian la reciente migración africana hacia y por América Latina desde distintos ángulos temáticos y disciplinarios. Sin embargo, reconocemos que cualquier estudio de un grupo particular de personas migrantes en un contexto específico corre el riesgo de esencialismo. En los estudios de migración, la perspectiva transnacional ha exigido cuestionar el llamado nacionalismo metodológico, lo cual asume la "naturaleza" de ciertas categorías de investigación y análisis basadas en los límites del Estado-nación, en particular, la nacionalidad y la etnicidad de las personas migrantes y su importancia en la vida de ellas (Amelina, Faist, 2012) ${ }^{2}$. Siguiendo esta línea, incluso la categoría misma de migrante requiere problematización, para evitar lo que Allison Hui llama la "excepcionalización del migrante" (Hui, 2016, p. 74-76, nuestra traducción). Puesto que hablar de personas migrantes necesariamente refleja delineaciones impuestas y supone la continua relevancia de prácticas e identidades de migración en la vida del grupo denominado "migrante". Al mismo tiempo, no podemos ignorar las transformaciones recientes acerca de las migraciones en el continente.

Entonces, ¿cómo podríamos responder a las dinámicas recientes de la migración africana en América Latina sin esencializar la categoría de 2 De forma comparable, se podría problematizar la categoría de "América Latina". Sobe una
geografía crítica de la región, ver Finn y Hanson (2017) y Zaragocin Carvajal et alii (2018). 
migrante africano? En esta contribución, utilizamos tres estrategias para evitar tal esencialismo e, igual de importante, para ir más allá de una simultanea racialización y victimización de las personas migrantes. En primer lugar, nos basamos en una exploración de la agencia humana, viendo cómo y cuándo diferentes experiencias y prácticas relacionadas al ser migrante, a cierta nacionalidad, etnicidad, religión, entre otros, juegan un papel en la vida diaria. Además, enfatizamos que estas experiencias y prácticas podrían ser compartidas con otros grupos sociales. En segundo lugar, tomamos en cuenta la posibilidad de una existencia transnacional de las personas migrantes y sus estrategias de movilidad, sin embargo, sin olvidarnos del contexto de control migratorio y cómo este contexto puede dificultar dicha existencia. En tercer lugar, y por último, mantenemos que nuestro enfoque en el haciendolugar nos ayuda a evitar esencialismos de origen y destino, de exclusión e inclusión, de movilidad e inmovilidad. Como podemos ver en la discusión que sigue, el lugar es un nodo relacional. No es fijo; sino obtiene significado y es producido de manera continua por sus habitantes, incluso aquellos/-as que lo habitan temporalmente. Tal proceso de haciendo-lugar es el enfoque principal de esta contribución.

\section{El panorama de la migración africana en América Latina}

En esta sección ofrecemos algunas características de la actual migración africana en América Latina, tomando en cuenta el legado de la larga historia de trata transatlántica de personas esclavizadas, así como la consiguiente exclusión social de la población afrodescendiente en los países de la región. Respecto a la actual migración africana, también señalamos que, evidentemente, el grupo de migrantes africanos es diverso, al igual que la región latinoamericana y su atención a esta migración. Sin embargo, hay ciertas condiciones que comparten los países de la región. Entre estas condiciones están una infraestructura migratoria ya existente, por un lado. Por otro lado, se refiere a esta migración muchas veces con un idioma de "crisis", representándola como una movilidad afuera de control, así a veces legitimando la violencia estatal hacia las personas migrantes (Álvarez Velasco, 2016). Además, se destacan la falta de capacidad institucional y de recursos humanos y económicos, sobre todo cuando las personas migrantes llegan en grupos grandes o se encuentran "varadas" en las fronteras; la difícil estimación estadística del fenómeno debido a su irregularidad; la falta, en gran parte, de cooperación entre países latinoamericanos y la falta de relaciones diplomáticas con países de origen; la falta de familiaridad con la región en términos de idioma, topografía, e instituciones de las personas migrantes por un lado, y por otro, la cuestión de cómo atender a una migración tan diversa (FLACSO, 2011, p. 35; GS/OAS, IOM, 2016, p. 60). Con estas condiciones, los diferentes gobiernos de la región 
se ven obligados a enfrentar múltiples preocupaciones en conjunto y establecer agendas comunes para atender a esta migración (Freier, 2013, p. 17).

Para una aproximación al contexto latinoamericano, nos basamos en los países de destino y tránsito actualmente más comunes. En América del Sur se ha notado un aumento de ingreso de personas migrantes africanas en los años 2000, con Argentina y Brasil siendo los destinos más buscados (OIM, 2012, p. 34). Hablando de la más reciente migración no-esclavizada africana hacia Brasil y Argentina, se destacan algunos momentos de incrementada llegada de migrantes. En el caso argentino, se notó una creciente inmigración desde Senegal, Nigeria, Ghana, la República Democrática del Congo y el Camerún, entre otros, a partir de la segunda mitad de los años 1990, con la migración senegalesa siendo la más numerosa (Zubrzycki, 2012). Mientras que Argentina no entretiene muchas relaciones diplomáticas y otras con países africanos, Brasil ha establecido una más estrecha colaboración, en términos técnicos, comerciales y culturales, con Angola, entre otros (Patarra, Fernandes, 2011). Desde este país Ilegaron numerosos migrantes a Brasil, sobre todo en la época de la independencia angolana en 1975, así como a partir de los 1980, muchas veces huyendo los conflictos internos del país (Baptista, 2007; Marcelino, Cerrutti, 2012; Patarra, Fernandes, 2011). Además de la llegada de migrantes de varios otros países africanos, otra vez se destaca la inmigración senegalesa hacia Brasil que se intensificó a partir de 2012 (Redin, Bittencourt Minchola, 2015, p. 202) y que, aquí también, ha captado atención en el ámbito académico (Merlotti Herédia, 2015; Tedesco, Kleidermacher, 2017).

Argentina y Brasil se han destacado como destinos migratorios populares por su relativa estabilidad económica y política. En el caso de Brasil, también importó su incrementada visibilidad internacional debida a la organización de grandes eventos deportivos como la copa mundial del fútbol en el 2014 y los juegos olímpicos en el 2016 (Heil, 2017; Espiro, 2017). No menos importante, a diferencia de las cada vez más restrictivas políticas migratorias europeas, ciertos países en América del Sur, como Ecuador, Argentina y Brasil, han liberalizado sus políticas migratorias o, por lo menos, adoptado un discurso relativamente liberal con respecto a la migración desde el inicio del siglo XXI (Acosta Arcarazo, Freier, 2015), lo que contribuyó al establecimiento de nuevas rutas migratorias hacia el continente (Zubrzycki, Agnelli, 2009; Maffia, 2010; Marcelino, Cerrutti, 2012).

Mientras que los recientes cambios de gobierno en los países sudamericanos señalan una retoma de discursos más restrictivos y securitarios con relación a las migraciones (Álvarez Velasco, 2016), Argentina, Ecuador y Brasil pasaron por una época en la cual se adoptó un fuerte discurso de derechos humanos. Sin embargo, los/las investigadores trabajando en el área de las migraciones señalaron con anterioridad las deficiencias de los 
programas de regularización realizadas en estos países, así como han mostrado qué dificultades tienen las personas migrantes de países africanos, asiáticos y caribeños, con la regularización de su título de residencia en dichos países (Acosta Arcarazo, Freier, 2015). Para el caso argentino, Eduardo Domenech escribió de "políticas de control con rostro humano" (Domenech, 2013), demostrando que las políticas basadas en un discurso de derechos humanos perseguían el mismo objetivo de controlar a las migraciones internacionales que las políticas abiertamente restrictivas. Por supuesto, las personas migrantes adaptan sus rutas a los cambios frecuentes en la región, en términos de legislación, de control fronterizo, de restricciones que se producen de un lado mientras que se abren nuevas oportunidades del otro. El caso de la migración senegalesa, una de las migraciones africanas recientes más bien estudiadas, demuestra que ya al llegar a América del Sur, muchas personas migrantes cruzan varios países de la región para entrar, por vía terrestre, y frecuentemente de manera "irregular", a Argentina o a Brasil (Zubrzycki, 2012; Merlotti Herédia, Santos Gonçalves, 2017)3.

La actual migración africana, y especialmente senegalesa, hacia Brasil y Argentina ha sido investigada en términos de las redes transnacionales y locales, así como las organizaciones religiosas y no-religiosas de las personas migrantes (Zubrzycki, Sánchez Alvarado, 2016; Maffia et alii, 2018; Heil, 2018; Romero, 2017; Reiffen, 2017). También han captado la atención sus prácticas laborales y su inserción en el mercado de trabajo local. Se han estudiado, entre otros, sus actividades relacionadas a la venta ambulante u otros trabajos comerciales (Zubrzycki, Agnelli, 2009; Zubrzycki et alii, 2010; Mocellin, 2017), o a los frigoríficos en el sur de Brasil donde se produce carne de forma halal y cuales constituyen, por ende, una posibilidad de empleo para migrantes musulmanes (Tedesco, 2017). No se deben olvidar aquellas personas migrantes africanas que vienen a dedicarse a estudios universitarios (Mendes De Gusmão, 2011; Baptista, 2007; Patarra, Fernandes, 2011). Otro campo de estudio relevante se demarca en el área de la representación de dichas personas migrantes en la prensa local y su percepción en la sociedad brasileña o argentina, percepciones y representaciones que, muchas veces, son exotizantes y hasta racistas (Morales, 2017; Morales, Kleidermacher, 2015; Sacramento, Machado, 2015; Rangel, 2015). Por supuesto, también importa subrayar cómo las personas migrantes se organizan para enfrentar prácticas discriminatorias (Espiro et alii, 2016) y cómo sus organizaciones se articulan

\footnotetext{
También hay migrantes senegaleses quienes entran de manera regular y directa por avión a sus países de destino. Sin embargo, en el caso de la migración senegalesa hacia Argentina, se presenta el problema de que la próxima representación diplomática accesible de Argentina en el continente africano se encuentra en Abuja, Nigeria. Los que logran obtener una visa en Abuja pueden entrar de manera regular a Argentina (Kleidermacher, 2013, p. 118).
} 
con movimientos locales, por ejemplo, con el movimiento afrodescendiente en Argentina (Maffia, Zubrzycki, 2014).

Se destaca claramente que, en muchos aspectos, las personas migrantes africanas se han insertado en los contextos locales en Argentina y en Brasil. Sin embargo, para muchas, América del Sur no es un punto final de un viaje planificado en detalle. Para algunas, siempre ha sido una etapa en un viaje más largo hacia América del Norte (Freier, 2013); para otras, la actual crisis económica y los cambios políticos en la región son razones por las cuales se ponen otra vez en movimiento, buscando mejores condiciones de vida afuera de la región.

Siguiendo el viaje a través de América del Sur, los primeros países más transitados son Ecuador y Colombia. En comparación con el contexto argentino y brasileño, todavía existen menos estudios sobre la reciente migración africana en esta zona. Ecuador es reconocido por ser una "puerta sudamericana" (Álvarez Velasco, 2016, p. 32) por sus normas migratorias relativamente abiertas. Tales normas, inspiradas por una "política de ciudadanía universal", han convertido el país en puerta de entrada, sala de espera e incluso destino para ciertos grupos de migrantes. Para las personas migrantes africanas, Ecuador se ha vuelto sobre todo un país de entrada y de tránsito, un "nodo articulador" (Ménard Marleau, 2017) para proseguir el viaje hacia el Cono Sur o hacia América del Norte. En cambio, en Colombia no existe una política global para la migración, en la práctica resultando en un énfasis en control y la negación de derechos y atención (Echeverri Zuluaga, Acevedo Sáenz, 2018). Aunque tanto en Ecuador como en Colombia, recientemente las normas migratorias se han puesto más restrictivas, el Estado colombiano parece estar más preocupado de ser utilizado como país de tránsito y del supuesto abuso de solicitudes de refugio. Además, vincula de manera más directa el tránsito de personas con la corrupción y el crimen organizado, particularmente el tráfico de drogas (Salazar et alii, 2013).

Una vez pasado Ecuador y Colombia, la peligrosa selva de Darién marca la entrada a la región centroamericana, una región de tránsito "por ser ese estrecho 'puente' de tierra que conecta el Sur con el Norte de América" (SJM-CR, RJM-CA, 2017, p. 6). Aunque reconoce que la cantidad de personas migrantes africanas en tránsito por la región está creciendo, Rocha (2016) insiste que la migración africana a través de Centroamérica no es un fenómeno nuevo. Más bien, ha sido invisibilizada por los gobiernos centroamericanos. Recientemente, esta migración se volvió más visible en el marco de las así llamadas "crisis migratorias", resultando del cierre (temporal) de varias fronteras después de 2015 que dejó a diferentes grupos de personas migrantes varadas y que requirió una respuesta formal (SJM-CR, RJM-CA, 
$2017)^{4}$. Entre estos grupos se marcó la presencia de personas africanas, que así empezaron a generar cada vez más atención en las noticias (Brenes, 2016; Castillo, 2015; Nicolau, 2016) y en las publicaciones de varias instituciones como la Organización de los Estados Americanos y la Organización Internacional para las Migraciones (GS/OAS, IOM, 2016) y el Servicio Jesuita para Migrantes (SJM-CR, RJM-CA, 2017). También existen algunos estudios exploratorios sobre la migración africana por esta zona (Drotbohm, Winters, 2018; FLACSO, 2011; Mora Izaguirre, 2017; Winters, Mora Izaguirre, 2019). Sin embargo, todavía hay muy poco conocimiento profundo del fenómeno. A través de las publicaciones mencionadas, se conoce los países principales de tránsito en la región: Panamá, Costa Rica, Nicaragua, Honduras y Guatemala - aunque las rutas son muy cambiantes debido a razones geopolíticas (véase Mata Blanco, 2017). También se estima que han pasado miles de personas migrantes africanas por año después de 2015, y se sabe que estas personas enfrentaron tanto abuso y peligro como asistencia humanitaria, tanto fronteras cerradas como permisos de paso. Lo que también se ha establecido, es que la gran mayoría de estas personas migrantes ven a Centroamérica como una zona de tránsito, y son vistas como personas en tránsito, aunque a veces varadas, por los gobiernos, instituciones y habitantes centroamericanos.

En conjunto, esta situación se extiende a México, históricamente conectado a la región centroamericana por la migración (Sandoval Garcia, 2015). México, aparte de ser el origen de muchas personas migrantes en los Estados Unidos, también es un país clave de tránsito (y cada vez más de destino), en primer lugar, para personas de Centroamérica, pero también de Sudamérica y de otros continentes. Sin embargo, México tiene la dudosa fama de ser un país muy violento para las personas migrantes en tránsito, desde las extorciones de organizaciones criminales y la impunidad hasta la externalización de fronteras, exigida por los Estados Unidos, la cual lleva a estas personas a tomar rutas cada vez más peligrosas (Aikin, Anaya Muñoz, 2013; Álvarez Velazco, 2016; Casillas, 2008; Vogt, 2017; Winters, Mora Izaguirre, 2019). Se podría imaginar que la relativa visibilidad de las personas africanas y su desconocimiento de la región les hace un blanco aún más fácil para vivir violencia en camino (FLACSO, 2011, p. 6; véase De León, 2015, p. 130).

Al mismo tiempo, como el conocimiento de su situación es muy limitado, hay que procurar no sacar conclusiones precipitadas. Además, como señala Mbuyi Kabunda Badi (2017) para el caso argentino, hay que evitar la victimización de las personas migrantes. No solo por su agencia humana,

\footnotetext{
${ }_{4}$ En este cuaderno de reflexión, el Servicio Jesuita para Migrantes describe de manera detallada las "crisis migratorias", los "flujos controlados", y los "tapones" en la ruta de esta migración, tal como las instituciones que se ocuparon de administrar acampamentos, albergues o centros de atención/detención de migrantes.
} 
sino también por los tambaleantes contextos geopolíticos y humanitarios que influyen en sus circunstancias y experiencias (véase Winters, 2018). Esta matización nos lleva a identificar ciertos huecos en los estudios sobre la migración africana en la región. Como señalamos arriba, se ha analizado más en profundidad la migración africana, sobre todo senegalesa, en el contexto argentino y brasileño. Sin embargo, fuera de estos estudios y particularmente en contextos menos estudiados prevalece un enfoque en supuestas situaciones de "crisis", tanto una crisis de zonas de tránsito que no pueden absorber y procesar a esta migración, como una crisis de las mismas personas migrantes, por ser víctimas de pobreza y violencia en sus países de origen y de abuso a mano de traficantes y pandillas en el camino. Además, como mencionado arriba, se asuma cierto nivel de rechazo e incluso xenofobia en las poblaciones locales. Surge, pues, la pregunta de qué pasaría si nos enfocáramos en inserciones y apropiaciones locales, a pesar de las condiciones estructurales de marginalización existentes, y a pesar de la presencia temporal de las personas migrantes. Por lo tanto, proponemos para este dossier una perspectiva de haciendo-lugar.

\section{Consideraciones teóricas}

\section{Regímenes de movilidad y la industria de la migración}

En el ámbito político y económico, así como en las ciencias sociales, se ha demarcado, en estos últimos años, un gran interés en la movilidad. A pesar de una creciente circulación global de servicios y productos, en paralelo crecen las prácticas y los discursos estatales que implementan medidas restrictivas a la movilidad humana, principalmente en las fronteras entre países y los controles fronterizos selectivos. Al mismo tiempo, en el ámbito académico, se ha empezado a analizar los movimientos de personas y cosas a través del lente analítico de la movilidad. Mientras que la movilidad fue celebrada muchas veces como "libertad", opuesta a una menos apreciada inmovilidad (Miglierina, Pereyra Iraola, 2018), nunca ha sido voluntaria ni libertadora para todos/-as, sino muchas veces enmarcada por necesidades económicas o por violencia. Tampoco se ha vuelto accesible para todos/-as, como comenta el antropólogo Noel Salazar:

las movilidades se han convertido en un nuevo factor de estratificación, produciendo una jerarquía global de movimientos. A medida que más personas cruzan las fronteras físicas y sociales, las autoridades e instituciones recurren a diversas infraestructuras y regímenes de movilidad para mantener el control. (Salazar, 2018, p. 10)

Al mismo tiempo, Daniela Miglierina y Victoria Pereyra Iraola (2018) nos conciencian sobre la problemática evaluación negativa de la inmovilidad y la 
necesidad de explorar la pluralidad y autonomía inherentes en la inmovilidad. Se refieren, entre otros, a David Bissel, quien analiza la espera como un momento de actividad y de potencialidad, en vez de conceptualizarla como un estado de parálisis o de estasis (Miglierina, Pereyra Iraola, 2018, p. 85-86). Para evitar una normalización de la movilidad así como de la inmovilidad $y$, al mismo tiempo, hacer surgir las relaciones de poder que posibilitan la movilidad de algunos mientras impiden la movilidad de otros ${ }^{5}$, Nina Glick Schiller y Noel Salazar proponen el término "regímenes de movilidad" (Glick Schiller, Salazar, 2013, p. 6).

Según Sofía Zaragocin Carvajal y sus colegas (2018, p. 22), una exploración crítica de la movilidad permite "re-historizar y re-politizar la migración latinoamericana y comprenderla como un proceso que articula y transforma el espacio a nivel multiescalar". En América del Sur, como ha observado el sociólogo Eduardo Domenech (2013; Domenech et alii, 2016), en los últimos años los regímenes de movilidad se han destacado por un discurso de derechos humanos frente a las migraciones internacionales con nuevas medidas de control y de vigilancia, materializándose en omisiones selectivas de visados, rechazos de ingreso en ciertas fronteras, y el registro de datos biométricos. Según las instituciones estatales y no-estatales que las ponen en práctica, estas medidas persiguen el objetivo de impedir la "migración irregular" o el "tráfico de personas". Tal perspectiva de gobernabilidad migratoria (migration management), según Domenech (2013), se complementa con la securitización de las migraciones. Una situación semejante se presenta en México y Centroamérica, en particular, a través del proceso de la externalización de fronteras desde los Estados Unidos hacia el sur (Winters, Mora Izaguirre, 2019). La articulación de prácticas de gobernabilidad migratoria, de securitización y del discurso enfocado en la defensa de derechos humanos, o, como lo ponen Domenech, Araujo y Torrano, el enfoque en la "'seguridad estatal' como en la 'seguridad humana', invita a pensar sobre la formación de un singular régimen de control de la migración, en particular de la migración 'irregular' o 'indocumentada'" (Domenech et alii, 2016, p. 2).

Mientras están surgiendo nuevas prácticas de control, también persiste la porosidad de ciertas fronteras, en el ámbito sudamericano como centroamericano y mexicano. En donde coexisten el control como imaginación ideal y su falta real, las personas migrantes buscan y encuentran diferentes caminos para llegar a sus destinos. Al mismo tiempo, aquellas que eluden los controles fronterizos son ilegalizadas, y sus movimientos cada vez más comercializados. Según SJM-CR y RJM-CA (2017, p. 7), "un aspecto que merece

5 Véase también el concepto de la "geometría del poder de la movilidad" (power-geometry of mobility) elaborado por la geógrafa Doreen Massey (1993). 
atención es el hecho de que estas dinámicas migratorias transcontinentales son movidas en gran medida a través de una poderosa y organizada red de tráfico de personas". Se nota, entonces, que la fricción entre la movilidad humana y las barreras geopolíticas a tal movilidad contribuyen al establecimiento de una "industria de la migración" (Casillas, 2008; Gammeltoft-Hansen, Sørensen, 2013; Hernández-León, 2012). Aunque este concepto no es nuevo, en los últimos años ha sido desarrollado de manera más aplicada, sosteniendo que esta industria juega un papel importante en la volatilidad de las trayectorias migratorias. Según el sociólogo Rubén Hernández-León (2012), la industria de la migración consiste en las infraestructuras de actores y actividades, tanto formales como informales, que con fines de lucro facilitan la migración. Aunque este autor reconoce y enfatiza las relaciones de la industria de la migración con actores y actividades no-empresariales, como los/las agentes de gobierno, los/ las trabajadores y voluntarios/-as de las ONG, y las mismas redes de migrantes, él no les considera como parte de la misma industria. En cambio, Hans Gammeltoft-Hansen y Ninna Nyberg Sørensen (2013) definen la industria de la migración como compuesta por todos/-as los/las actores y actividades que facilitan e impiden la migración, tanto con fines de lucro como con otros fines no-económicos, y los vínculos entre estos/-as actores y actividades. La ventaja de verla de esta manera más holística es que ilumina cómo estos/-as actores y actividades no están simplemente vinculados en el conjunto que es la industria, sino también cómo que se alimentan entre ellos (Vogt, 2016). Por ejemplo, la tensión entre la seguridad y la movilidad genera ganancias y nuevos paisajes tecnológicos. Además, produce diferentes posibilidades e imposibilidades de migración, lo cual resulta en trayectorias de movilidad e inmovilidad cada vez más complejas (Mainwaring, Brigden, 2016).

\section{Haciendo-lugar y haciendo-Lugar}

En su monografía "Being-Here", la antropóloga Annika Lems (2018) revisa cómo se transformó la concepción y el papel asignado al lugar en su disciplina. Mientras que, por mucho tiempo, las culturas fueron consideradas fijadas a un territorio particular, desde los años 1980, la supuesta estabilidad y el arraigo de cultura e identidad fueron criticado desde una perspectiva social-constructivista. Esta crítica, según Lems, llevó a la tendencia de priorizar movimiento, fluidez, e finalmente, de hacer de "desplazamiento [...] el nuevo tropo a través del cual los antropólogos llegaron a mirar al mundo" (nuestra traducción, Lems, 2018, p. 14). Frente a este enfoque en la movilidad, el "desarraigo" y la mundialización, Lems, así como, ya antes, el antropólogo Arturo Escobar (2000), critican un cierto abismo que se haya desarrollado entre la teoría y la experiencia de los seres humanos. Ambos/-as argumentan que el "lugar [...] continúa siendo importante en la vida de la mayoría de las 
personas, quizás para todas" (Escobar, 2000, p. 113). La experiencia del ser humano, sostiene el antropólogo Tim Ingold, invita a re-establecer la noción del lugar contra una conceptualización vacía del espacio. Pensando en un mapa visto desde arriba, Ingold, en su imaginación, adopta la perspectiva del caminante (wayfarer), para quien "el mundo no se presenta como una superficie a ser recorrida. En sus movimientos, él enhebra su camino por este mundo más que encaminarse a través de él, de un punto a otro. Por supuesto el caminante es un ser terrestre, y debe forzosamente viajar sobre la tierra" (Ingold, 2015, p. 19, énfasis en original).

En los estudios migratorios, destacamos un desarrollo parecido, desde una celebración de flujos a una re-apreciación de lo anclado. Aunque el enfoque al transnacionalismo de las personas migrantes ha llevado a acentuar la volatilidad y la "dimensión desterritorializada" (Tapia Ladino, 2015, p. 198) de las experiencias, prácticas y relaciones sociales, varios/-as autores han enfatizado la importancia de considerar cómo estas dinámicas transnacionales están situadas en lugares específicos, incluso más allá de origen y destino, con sus propias limitaciones y oportunidades (Guarnizo, Smith, 1998; Levitt, De La Dehesa, 2017; Olwig, 2003). Como escribe Sinatti (2008):

Las prácticas transnacionales de los inmigrantes contemporáneos [...] deben percibirse como marcadamente emplazadas. En otras palabras, en lugar de constituir un mero flujo de personas, recursos e imágenes itinerantes y en circulación, el transnacionalismo está presente en los lugares concretos en los que los inmigrantes viven sus vidas y realizan sus prácticas itinerantes. (Sinatti, 2008, p. 106)

Aunque las trayectorias de las personas migrantes sean cambiantes y volátiles y que siempre se alternen momentos de movimiento con momentos de inmovilidad, estas personas nunca dejan de hacer lugar de una manera u otra en su camino. Así que no son únicamente los lugares de partida, los lugares de nacimiento, o nociones de ser "nativo", que expresan la importancia que pueda tener el lugar para las personas. Esto ya observó la antropóloga Liisa Malkki en los años 1980, cuando estudió el establecimiento de personas refugiadas burundés en Tanzania. Más bien, refiriéndose a desplazamiento, la autora sostiene cómo concepciones fijas de lugar velan, más que aclaran, "la multiplicidad de apegos [attachment] que entablan las personas con los lugares, viviendo en ellos, memorándolos, e imaginándolos" (Malkki, 1992, p. 38, nuestra traducción). Según la autora, la percepción del vínculo entre las personas y sus lugares de origen como "natural" conduce a una interpretación del desplazamiento como estado patológico, una deviación del orden nacional naturalizado del mundo. Esto se expresa en la noción botánica, en este contexto aplicada a personas, de "desarraigo" (uprootedness, Malkki, 1992, p. 31-32). En cambio, el apego múltiple y subjetivo, muchas veces afectivo, 
que desarrollan las personas con los lugares lleva a una percepción del vínculo entre persona y lugar que no es preexistente o natural, como suponen los términos "arraigo" o "desarraigo", sino que se desarrolla con el tiempo (Aguilar Diaz, 2012). Eso implica también que el desarrollo de un vínculo con un lugar específico no necesariamente excluye que esta persona prosiga su trayecto, o que produzca un apego con distintos lugares por los cuales pasa en su camino. Incluso cuando personas migrantes quedan "varadas" en el viaje, su presencia temporal no descarta la posibilidad de varios tipos de inserciones y apropiaciones locales a través de la participación en alguna comunidad religiosa, el seguir clases de español y el trabajo informal en zonas fronterizas, entre otros (Drotbohm, Winters, 2018).

Bien entendido, en toda su crítica, los/las autores citados/-as arriba no exigen un retorno a un concepto esencialista del lugar. Para re-evaluar al lugar, re-apreciar lo específico, pero imaginándolo más abierto, nos basamos en los conceptos de espacio y de lugar formulados por la geógrafa Doreen Massey. Escribiendo desde una posición de responsabilidad política y social en un mundo desigual, Massey sostiene que

si el espacio no es simplemente la suma de territorios sinó [sic] una complejidad de relaciones (flujos y fronteras, territorios y vínculos) ello implica que 'un lugar', un territorio, no puede ser tampoco algo simple, cerrado y coherente. Al contrario, cada lugar es un nodo abierto de relaciones, una articulación, un entramado de flujos, influencias, intercambios, etc. [...] Es decir que la especificidad de cada lugar es el resultado de la mezcla distinta de todas las relaciones, prácticas, intercambios, etc. que se entrelazan dentro de este nodo y es producto también de lo que se desarrolle como resultado de este entrelazamiento. Es algo que yo he denominado 'un sentido global de lugar', un sentido global de lo local. (Massey, 2004, p. 78-79, énfasis en original) ${ }^{6}$

Un sentido global del lugar implica que "los lugares son nodos de relaciones entrelazadas" (p. 81), estos nodos (o nudos) se construyen a través de estas relaciones (o hilos, según Ingold, 2015, p. 14), incluyendo las relaciones establecidas por la migración.

Deberíamos ver, entonces, a los lugares como entidades dinámicas, siempre bajo construcción (Merriman, 2009, p. 10). Siguiendo a estos/-as autores, el lugar en sí mantiene una complejidad que, así pensamos nosotras,

\footnotetext{
6 Queremos señalar que, dentro de los debates sobre lugar y espacio surgieron otras definiciones. En una distinta línea de pensamiento, basándose en De Certeau, María Victoria Pita y sus colegas (2017), por ejemplo, definen al espacio como "vivido" y "producido por la práctica del lugar, de un mapa habitado", mientras que el lugar en sí "es una configuración instantánea de posiciones" (Pita et alii, 2017, p. 79). Posicionada entre las dos posturas se presenta la noción de "lugarización" de Paola Jirón. Jirón se refiere al moverse como "habitar en movimiento", un proceso que da sentido a los sitios que las personas transitan y los transforma, en la línea de pensamiento à la De Certeau, "en espacios significativos" (Jirón, 2018, p. 87).
} 
se tendría que manifestar también cuando hablamos del haciendo-lugar. Según Agnew y Duncan (2014 [1989], p. 2), el lugar combina tres dimensiones: su locación, su aspecto local, así como el sentido del lugar. Mientras que la locación refiere a su "existencia material que se corresponde a un dónde" (Aguilar Díaz, 2012, p. 123), su aspecto local describe el ambiente material que significa el lugar para las relaciones sociales. El sentido del lugar, como último, se refiere a la identificación con el lugar, al apego que las personas desarrollan con los lugares, por ende, a una dimensión afectiva. Agnew y Duncan (2014 [1989]) sostienen que estas tres dimensiones son complementarias, una opinión también compartida por Lems (2018, p. 108) y ofreciendo claves para refinar el haciendo-lugar. Como hemos visto más arriba, la distribución de poder y los regímenes políticos migratorios influyen sobre las posibilidades que tienen las personas para estar móvil o inmóvil, para quedarse y vincularse con un lugar. En relación a las prácticas concretas de las cuales consiste el haciendolugar, proponemos distinguir dos conjuntos de prácticas. Primero, las que efectúan un cambio en el aspecto material del lugar, las huellas que dejan las personas cuando atraviesan o se establecen en un lugar, es decir, todas las prácticas que implican la construcción del lugar. Tales prácticas ponen énfasis en lo duradero, literalmente en el sustantivo, el "haciendo-Lugar". Segundo, llamamos la atención otra vez a la dimensión afectiva del haciendo-lugar, el sentirse apegado a un lugar, el sentirse "en casa" y el identificarse con un lugar. Este último conjunto de experiencias y prácticas pone énfasis en el verbo, el "Haciendo-lugar". El Haciendo-lugar tanto como el haciendo-Lugar también juegan para personas migrantes "varadas" en el camino, porque, como sostienen Miglierina y Pereyra Iraola (2018), la espera y la inmovilidad no son estados inactivos. Mientras que no queremos volver a desenclavijar estas dimensiones del lugar, penosamente reunidas por otros investigadores, pensamos que contribuiría a la claridad del concepto de haciendo-lugar prestar atención a estos dos aspectos que, obviamente, son relacionados e interdependientes.

\section{Los artículos que componen este dossier temático}

Aparte de nuestra introducción, seis artículos componen el presente dossier, además complementado por una reflexión exploratoria sobre un trabajo de campo reciente. Todos los artículos retoman diferentes aspectos de las líneas de pensamiento planteadas arriba y las aplican a contextos diversos latinoamericanos. Sin embargo, cada artículo desarrolla su propio enfoque teórico, metodológico y empírico y, por lo tanto, puede ser leído por sí sólo.

La secuencia de los artículos mantiene una lógica que se mueve desde las circulaciones y las trayectorias a las inserciones locales. En el primer artículo del dossier, Rosana Baeninger, Natália Belmonte Demétrio y Jóice Domeniconi 
construyen un perfil sociodemográfico de las migraciones africanas a Brasil en el siglo XXI, así iluminando un espacio transnacional emergente. Basado en una perspectiva multi-escalar, que toma en cuenta tanto las transformaciones recientes en los mercados, conexiones y circulaciones internacionales como la posición y las características del estado de Brasil en ello, y combinando estadísticas de diferentes registros administrativos, las autoras elaboran un perfil sólido que nos ayuda a entender el contexto de las migraciones africanas recientes al continente americano.

En el siguiente artículo, Maria do Carmo dos Santos Gonçalves y Lucas Cé Sangalli concretizan esta perspectiva transnacional y circular, enfocándose en la re-construcción de las trayectorias migratorias de un migrante senegalés y un migrante ghanés. Basado en trabajo de campo etnográfico multisituado que toma como punto de partido un centro para migrantes en la ciudad brasileña Caxias do Sul, y utilizando el concepto de territorios de circulación, este artículo detalla dinámicas de poder y pertenencia en los desplazamientos, redes, movimientos e inmovilidades que viven estas personas migrantes. Enfatizando la agencia y las capacidades generativas de este grupo, este análisis complica categorías simplistas, estáticas y victimizantes de la migración africana.

En el tercer artículo, Maria Luz Espiro extiende un similar análisis a Argentina, presentando un artículo que detalla la complejidad de las trayectorias laborales (así como familiares y migratorias) de migrantes senegaleses en este país. Basado en trabajo de campo etnográfico en diferentes ciudades en Argentina y Senegal, este artículo aborda el rol de los agentes de control estatal, la prensa local, y las redes étnicas y transnacionales en las estrategias de las personas migrantes, estas últimas analizadas como el 'saber migrar' y el 'saber circular'. La autora reconoce y aclara cómo las trayectorias familiares y migratorias transnacionales están vinculadas con la situación laboral actual que viven las personas migrantes en Argentina, incluso con los esfuerzos de acción colectiva para mejorar dicha situación.

Explorando las dinámicas de esta acción colectiva más a la profundidad, en el cuarto artículo Sonia Raquel Voscoboinik y Bernarda Zubrzycki se enfocan en la agencia y el asociacionismo de migrantes senegaleses en la ciudad argentina de La Plata. Basado en un estudio multi-método, este trabajo busca entender cómo los vendedores senegaleses responden a situaciones de violencia por parte de las autoridades. Las autoras describen las negociaciones y desigualdades que son parte del surgimiento y funcionamiento de una asociación senegalesa local.

Vinculando el contexto laboral con lo religioso, en el quinto artículo Franziska Reiffen aborda experiencias de migrantes congoleñas y prácticas 
de haciendo-lugar en la ciudad brasileña de San Pablo. Explorando cómo estas migrantes (trans)forman lugares y redes en un contexto de desigualdad social, este trabajo etnográfico toma lugar en dos espacios claves para ellas, una peluquería y una parroquia evangélica. Una descripción meticulosa de estos espacios y sus dinámicas de trabajo, socialización y aprendizaje lleva a un análisis que acentúa la relación entre procesos de desplazamiento y de hacerse lugar, por mucho que sea precario.

En el sexto artículo del dossier, Margarita Rosa Gaviria Mejía y Marcele Scapin también exploran un espacio transnacional e íntimo a la vez, es decir, la tienda de un migrante senegalés y su esposa en una pequeña ciudad en Rio Grande do Sul, Brasil. A través de las dinámicas de esta tienda, este artículo aborda las identidades y prácticas religiosas y de género que influyen las experiencias migratorias de las personas migrantes. Las autoras abren su reflexión hacia afuera de la tienda, demostrando continuidades, así como diferencias con otros contextos de migración senegalesa. Sobre todo, resaltan cómo las relaciones de género en la comunidad de migrantes senegaleses se vinculan con aquellas en Senegal.

Finalmente, para complementar nuestro dossier, en su reflexión exploratoria sobre trabajo de campo en la provincia de Darién, Panamá, Nanneke Winters explora las posibilidades de haciendo-lugar en situaciones de tránsito. Panamá es visto como zona de paso, una visión fortalecida por su política de 'flujo controlado' que busca trasladar a las personas migrantes africanas lo más rápido posible a través del territorio panameño. Sin embargo, esta migración deja huellas y por lo tanto transforma el mismo espacio panameño, en términos de su infraestructura, su población y su paisaje.

En conjunto, estos artículos y reflexiones nos ayudan, no sólo a dar una impresión de la presente migración africana en América Latina y los estudios que se están realizando sobre ella, sino sobre todo a explorar los vínculos entre circulaciones, conexiones y lugares. Muestran cómo los entrelazamientos actuales de movilidad e inmovilidad, situados en la intersección de estructuras mercantiles y estatales, por un lado, y prácticas de personas migrantes por otro, Ilevan a ciertas experiencias de haciendo-Lugar y Haciendo-lugar que complican la visión dominante de crisis, esencialización y victimización sobre la migración africana. En nuestra opinión, las huellas y los apegos que exploran los artículos y reflexiones aquí nos ayudan a reconocer a la cara humana de esta migración.

Para concluir nuestra introducción, queremos llamar la atención sobre el desequilibrio geográfico de este dossier. A pesar de nuestros esfuerzos para reunir contribuciones desde una diversidad de países latinoamericanos, lamentablemente el conjunto de artículos presentado aquí refleja la relativa 
falta de estudios sobre la migración africana en países afuera del Cono Sur. Pensamos que este desequilibrio puede resultar, por una parte, del carácter más bien transitorio de esta migración en ciertas regiones (como Centroamérica), lo cual lo hace más difícil estudiar, y por otra, del énfasis en otras migraciones más numerosas (como la venezolana en Colombia y la centroamericana en México) que generan más atención. Sin embargo, aprovechamos este espacio para hacer una llamada a estudios de haciendolugar de las personas migrantes africanas en contextos más allá del Cono Sur, sobre todo en contextos no-urbanos y regiones fronterizas. Ampliar nuestro enfoque de esta forma nos ayudaría a contextualizar y entender mejor los diferentes compromisos de corta o larga duración de estas personas migrantes con la diversidad de lugares en los cuales habitan o que atraviesan en sus trayectorias por el continente latinoamericano.

\section{Referencias}

ACOSTA ARCARAZO, Diego; FREIER, Luisa Feline. Turning the Immigration Policy Paradox Upside Down? Populist Liberalism and Discursive Gaps in South America. International Migration Review, v. 49, n. 3, p. 659-696, 2015.

AGNEW, John A.; DUNCAN, James S. Introduction. In: AGNEW, John A.; DUNCAN, James S. (orgs.). The power of place. Bringing together geographical and sociological imaginations. London, New York: Routledge, 2014 (1989), p. 1-8.

AGUILAR DÍAZ, Miguel Ángel. Antropología urbana y lugar. Recorridos conceptuales. In: GIGLIA, Angela; SIGNORELLI, Amalia (orgs.). Nuevas topografías de la cultura. Ciudad de México: Universidad Autónoma Metropolitana, Juan Pablos Editor, 2012, p. 113-144.

AIKIN, Olga; ANAYA MUÑOZ, Alejandro. Crisis de derechos humanos de las personas migrantes en tránsito por México: Redes y presión transnacional. Foro Internacional, v. 53, n. 1, p. 143-181, 2013.

ÁLVAREZ VELASCO, Soledad. Control y violencia ante la migración irregularizada por la región. Seguridad y Sociedad, v. 6, n. 14, p. 30-38, 2016.

AMELINA, Anna; FAIST, Thomas. De-naturalizing the national in research methodologies: key concepts of transnational studies in migration. Ethnic and Racial Studies, v. 35, n. 10, p. 1707-1724, 2012.

BAPTISTA, Dulce Maria Tourinho. Migração na metrópole: o caso dos angolanos em São Paulo. Cadernos metrópole, n. 17, p. 103-118, 2007.

BRENES, Danny. África varada: la crisis migratoria en Paso Canoas (2016). La Nación, 24.04.2016. Disponible en: <https://www.nacion.com/el-pais/politica/ africa-varada-la-crisis-migratoria-en-paso-canoas/6E7JDFAIRRHWXBSQDIAGL HFY6I/story/>. Acceso en: 10.04.2019.

CASILLAS, Rodolfo. Las rutas de los centroamericanos por México, un ejercicio de caracterización, actores principales y complejidades. Migración y Desarrollo, n. 10, p. 157-174, 2008. 
CASTILLO, Diego. Policía desarticula banda internacional de tráfico de personas (2015). La Nación, 10.11.2015. Disponible en: <https://www.nacion.com/ sucesos/crimenes/policia-desarticula-banda-internacional-de-trafico-de-personas/ DQCWEC3NGBHNJIQHWZSTMHCXDQ/story/>. Acceso en: 10.04.2019.

DE LEÓN, Jason. The Land of Open Graves. Living and Dying on the Migrant Trail. Oakland: University of California Press, 2015.

DOMENECH, Eduardo. "Las migraciones son como el agua": Hacia la instauración de políticas de "control con rostro humano". La gobernabilidad migratoria en la Argentina. Polis [en ligne], n. 35, 2013. Disponible en: < http://polis.revues. org/9280>. Acceso en: 10.04.2019.

DOMENECH, Eduardo; ARAUJO, Sandra Gil; TORRANO, Andrea. Editorial. Pasado y presente de las políticas y prácticas de control y vigilancia de la migración en Sudamérica. Astrolabio Nueva Época, n. 17, p. 1-5, 2016.

DROTBOHM, Heike; WINTERS, Nanneke. Transnational Lives en Route: African Trajectories of Displacement and Emplacement across Central America. Working Papers of the Department of Anthropology and African Studies of the Johannes Gutenberg University Mainz, 175, 2018.

ECHEVERRI ZULUAGA, Jonathan; ACEVEDO SÁENZ, Liza. Pensando a través de la errancia: travesías y esperas de viajeros africanos en Quito y Dakar. Antípoda. Revista de Antropología y Arqueología, n. 32, p. 105-123, 2018.

ESCOBAR, Arturo. El lugar de la naturaleza y la naturaleza del lugar: ¿globalización o postdesarrollo? In: LANDER, Edgardo (org.) La colonialidad del saber: eurocentrismo y ciencias sociales. Perspectivas latinoamericanas. Buenos Aires: CLACSO, 2000, p. 113-144.

ESCOBAR, Arturo. Territorios de diferencia. Lugar, movimientos, vida, redes. Popayán: Envión, 2010.

ESPIRO, María Luz; VOSCOBOINIK, Sonia; ZUBRZYCKI, Bernarda. Enfrentando el racismo institucional. Análisis de dos casos de migrantes senegaleses en Argentina (2012-2016). REMHU, Revista Interdisciplinar da Mobilidade Humana, v. XXIV, n. 48, p. 63-78, 2016.

ESPIRO, María Luz. Senegaleses entre la Argentina y el sur de Brasil: etnografía de la movilidad regional y la alternancia laboral entre venta ambulante e industria. In: MAFFIA, Marta M; ZUBRYZCKI, Bernarda (orgs.). Africanos y afrodescendientes en la Argentina. Prácticas, representaciones, narrativas y memorias. Buenos Aires: Biblos, 2017, p. 41-60.

FREIER, Luisa Feline. Introducción. In: Migrantes extracontinentales en América del Sur: Estudio de casos. Cuadernos Migratorios n. 5. Buenos Aires: Organización Internacional para las Migraciones, 2013.

FLACSO. Diagnóstico sobre la situación actual, tendencias y necesidades de protección y asistencia de las personas migrantes y refugiadas extracontinentales en México y América Central. San Jose: Facultad Latinoamericana de Ciencias Sociales (FLACSO), 2011. 
FINN, John C.; HANSON, Anne-Marie. Critical Geographies in Latin America. Journal of Latin American Geography, v. 16, n. 1, p. 1-15, 2017.

GAMMELTOFT-HANSEN, Thomas; SØRENSEN, Ninna (orgs.). The Migration Industry and the Commercialization of International Migration. London: Routledge, 2013.

GLICK SCHILLER, Nina; SALAZAR, Noel. Regimes of Mobility Across the Globe. Journal of Ethnic and Migration Studies, v. 39, n. 2, p. 183-200, 2012.

GS/OAS; IOM. Regional Report: Irregular Migration Flows to the Americas from Africa, Asia, and the Caribbean. Washington: The General Secretariat of the Organization of American States (GS/OAS) and the International Organization for Migration (IOM), 2016.

GUARNIZO, Luis E.; SMITH, Michael P. The locations of transnationalism. In: SMITH, Michael P.; GUARNIZO, Luis E. (orgs.). Transnationalism from Below. New Brunswick: Transaction Publishers, 1998, p. 3-31.

HEIL, Tilmann. Perder, só perder. Vendedores senegaleses durante os jogos olímpicos no Rio de Janeiro. In: TEDESCO, João Carlos; KLEIDERMACHER, Gisele (orgs.). Imigração senegalesa no Brasil e na Argentina: multiplos olhares. Porto Alegre: Est Edições, 2017, p. 229-254.

HEIL, Tilmann. Uma infraestrutura muçulmana de chegada no Rio de Janeiro. REMHU, Revista Interdisciplinar da Mobilidade Humana, v. 26, n. 52, p. 111129, 2018.

HERNÁNDEZ-LEON, Rubén. La industria de la migración en el sistema migratorio México-Estados Unidos. Trace, n. 61, p. 41-61, 2012.

HUI, Allison. The Boundaries of Interdisciplinary Fields: Temporalities Shaping the Past and Future of Dialogue between Migration and Mobilities Research. Mobilities, v. 11, n. 1, p. 66-82, 2016.

INGOLD, Tim. Contra el espacio: lugar, movimiento, conocimiento. Revista Latinoamericana de Políticas y Acción Pública, v. 2, n. 2, p. 9-26, 2015.

JIRÓN, Paola. Lugarización en movimiento. In: ZUNINO SINGH, Dhan; GIUCCI, Guillermo; JIRÓN, Paola (orgs.). Términos clave para los estudios de movilidad en América Latina. Buenos Aires: Biblos, 2018, p. 87-93.

KABUNDA BADI, Mbuyi. Prólogo. In: MAFFIA, Marta M.; ZUBRZYCKI, Bernarda (orgs.). Africanos y afrodescendientes en la Argentina. Prácticas, representaciones, narrativas y memorias. Buenos Aires: Biblos, 2017, p. 9-13.

KLEIDERMACHER, Giselle. Entre cofradías y venta ambulante: una caracterización de la inmigración senegalesa en Buenos Aires. Cuadernos de antropología social, n. 38, p. 109-130, 2013.

LEMS, Annika. Being-Here. Placemaking in a World of Movement. New York, Oxford: Berghahn, 2018.

LEVITT, Peggy; DE LA DEHESA, Rafael. Rethinking transnational migration and the re-definition of the state or what to do about (semi-) permanent impermanence. Ethnic and Racial Studies, v. 40, n. 9, p. 1520-1526, 2017. 
MAFFIA, Marta M. Una contribución al estudio de la nueva inmigración africana subsahariana en la Argentina. Cuadernos de Antropología Social, n. 31, p. 7-32, 2010.

MAFFIA, Marta M.; ZUBRZYCKI, Bernarda. Relaciones, significados y orientaciones hacia un accionar colectivo de los afrodescendientes y los africanos en la Argentina. In: TAMAGNO, Liliana E.; MAFFIA, Marta M. (orgs.). Indígenas, africanos y afrodescendientes en la Argentina. Convergencias, divergencias y desafíos. Buenos Aires: Biblos, 2014, p. 183-199.

MAFFIA, Marta M.; MONKEVICIUS, Paola; ESPIRO, Luz; VOSCOBOINIK, Sonia. Formas organizativas y liderazgo entre inmigrantes africanos subsaharianos recientes. Revista Migraciones Internacionales, Reflexiones desde Argentina. Buenos Aires: Organización Internacional para las Migraciones (OIM), p. 9-28, 2018.

MAINWARING, Cetta; BRIGDEN, Noelle. Beyond the Border: Clandestine Migration Journeys. Geopolitics, v. 21, n. 2, p. 243-262, 2016.

MALKKI, Liisa. National Geographic: The Rooting of Peoples and the Territorialization of National Identity among Scholars and Refugees. Cultural Anthropology, v. 7, n. 1, p. 24-44, 1992.

MARCELINO, Pedro F.; CERRUTTI, Marcela. Recent African immigration to South America: The cases of Argentina and Brazil in the regional context. In: PIZARRO, Jorge Martínez; FINARDI, Leandro Reboiras (orgs.). Development, institutional and policy aspects of international migration between Africa, Europe and Latin America and the Caribbean. Santiago de Chile: Economic Commission for Latin America and the Caribbean (ECLAC), 2012, p. 107-145.

MASSEY, Doreen. Power-geometry and a progressive sense of place. In: BIRD, Jon; CURTIS, Barry; PUTNAM, Tim; ROBERTSON, George; TICKNER, Lisa (orgs.). Mapping the futures. Local cultures, global change. London: Routledge, 1993, p. 60-70.

MASSEY, Doreen. Lugar, identidad y geografías de la responsabilidad en un mundo en proceso de globalización. Treballs de la Societat Catalana de Geografia, n. 57, p. 77-84, 2004.

MATA BLANCO, Esteban, E. Migrantes y traficantes de personas evaden Costa Rica mediante rutas marítimas hacia Guatemala y México (2017). La Nación, Cidade, 13.05.2017. Disponible en: < https://www.nacion.com/el-pais/politica/migrantesy-traficantes-de-personas-evaden-costa-rica-mediante-rutas-maritimas-haciaguatemala-y-mexico/DKRDGMXJNZGXXPEDLW7LOHU2VM/story/>. Acceso en: 10.04.2019.

MÉNARD MARLEAU, Andrée. Ecuador como nodo articulador de la migración senegalesa en América del Sur. Migración y Desarrollo, v. 15, n. 29, p. 31-50, 2017.

MENDES DE GUSMÃO, Neusa Maria. "Na Terra do Outro": presença e invisibilidade de estudantes africanos no Brasil, hoje. Dimensões, v. 26, p. 191-204, 2011.

MERLOTTI HERÉDIA, Vania Beatriz (org.). Migrações internacionais: O caso dos senegaleses no sul do Brasil. Caxias do Sul: Quatrilho Editora, 2015. 
MERLOTTI HERÉDIA, Vania Beatriz; SANTOS GONÇALVES, Maria do Carmo. Deslocamentos populacionais no sul do Brasil: o caso dos senegaleses. In: TEDESCO, João Carlos; KLEIDERMACHER, Gisele (orgs.). Imigração senegalesa no Brasil e na Argentina: multiplos olhares. Porto Alegre: Est Edições, 2017, p. 209-228.

MERRIMAN, Peter. Marc Augé on Space, Place and Non-Places. The Irish Journal of French Studies, n. 9, p. 9-29, 2009.

MIGLIERINA, Daniela; PEREYRA IRAOLA, Victoria. Inmovilidad. In: ZUNINO SINGH, Dhan; GIUCCI, Guillermo; JIRÓN, Paola (orgs.). Términos clave para los estudios de movilidad en América Latina. Buenos Aires: Biblos, 2018, p. 81-86.

MOCELLIN, Maria Clara. Deslocamentos e trabalho ambulante entre jovens senegaleses no Rio Grande do Sul. In: TEDESCO, João Carlos; KLEIDERMACHER, Gisele (orgs.). Imigração senegalesa no Brasil e na Argentina: multiplos olhares. Porto Alegre: Est Edições, 2017, p. 339-357.

MORA IZAGUIRRE, Cynthia. De Brasil hasta Canadá: el paso de los extracontinentales por Costa Rica. In: MORA IZAGUIRRE, Cynthia (org.). Migraciones en Costa Rica. Un fenómeno histórico y dinámico desde diversas perspectivas disciplinares. San José: FLACSO, 2017, p. 175-203.

MORALES, Orlando Gabriel; KLEIDERMACHER, Gisele. Representaciones de migrantes senegaleses en la sociedad porteña de Buenos Aires: apuntes sobre exotismo y exotización. Etnográfica, v. 19, n. 1, p. 29-50, 2015.

MORALES, Orlando Gabriel. Adscripciones y representaciones de migrantes africanos en sus interacciones cotidianas en las ciudades de Buenos Aires y La Plata. In: MAFFIA, Marta M.; ZUBRYZCKI, Bernarda (orgs.). Africanos y afrodescendientes en la Argentina. Prácticas, representaciones, narrativas y memorias. Buenos Aires: Biblos, 2017, p. 21-39.

NICOLAU, Lisa. Africans join the mass migration movement through Latin America (2016). Humanosphere, 16.09.2016. Disponible en: <http://www. humanosphere.org/basics/2016/09/africans-join-mass-migration-movement-latinamerica/>. Acceso en: 10.04.2019.

OIM. Panorama migratorio de América del Sur. Buenos Aires: Organización Internacional para las Migraciones (OIM), 2012.

OLWIG, Karen F. Transnational socio-cultural systems and ethnographic research: views from an extended field site. International Migration Review, v. 37, n. 3, p. 787-811, 2003.

PATARRA, Neide Lopes; FERNANDES, Duval. Brasil: pais de imigração? Revista Internacional em Língua Portuguesa. Migrações, n. 24, p. 65-96, 2011.

PITA, María Victoria; GÓMEZ, Joaquín Santiago; SKLIAR, Mariano. Historias mínimas. Apuntes para una etnografía del control contravencional y la gestión de ilegalismos en la Ciudad de Buenos Aires. In: PITA, María Victoria; PACECCA, María Inés (orgs.). Territorios de control policial. Gestión de ilegalismos en la Ciudad de Buenos Aires. Buenos Aires: Facultad de Filosofía y Letras, Universidad de Buenos Aires, 2017, p. 77-146. 
RANGEL, Larissa. Onde está a África no Brasil? Um retrato da recente imigração senegalesa sob o olhar da mídia brasileira. In: MERLOTTI HERÉDIA, Vania Beatriz (org.). Migrações internacionais: O caso dos senegaleses no sul do Brasil. Caxias do Sul: Quatrilho Editora, 2015, p. 67-92.

REDIN, Giuliana; BITTENCOURT MINCHOLA, Luís Augusto. Imigrantes senegaleses no Brasil: Tratamento jurídico e desafios para a garantia de direitos. In: MERLOTTI HERÉDIA, Vania Beatriz (org.). Migrações internacionais: O caso dos senegaleses no sul do Brasil. Caxias do Sul: Quatrilho Editora, 2015, p. 201-217.

REIFFEN, Franziska. ¿Casamancés, africano, negro? Negociación de identificaciones dentro de un grupo de mujeres migrantes en Buenos Aires. In: TEDESCO, João Carlos; KLEIDERMACHER, Gisele (orgs.). Imigração senegalesa no Brasil e na Argentina: multiplos olhares. Porto Alegre: Est Edições, 2017, p. 151-175.

ROCHA, José Luis. Miles de migrantes africanos en nuestras fronteras. Envío digital, n. 414, 2016. Disponible en: <http://www.envio.org.ni/articulo/5245>. Acceso en: 10.04.2019.

ROMERO, Fanny Longa. Islã, parentesco e ritual na irmandade religiosa Mouridiyya: percursos da etnografia no contexto da imigração de africanos senegaleses no Brasil. In: TEDESCO, João Carlos; KLEIDERMACHER, Gisele (orgs.). Imigração senegalesa no Brasil e na Argentina: multiplos olhares. Porto Alegre: Est Edições, 2017, p. 275-296.

SACRAMENTO, Igor, MACHADO, Izamara Bastos. A imigração como risco para a saúde: uma análise das representações do imigrante africano na cobertura da Folha de São Paulo sobre o ébola. Comunicação e Sociedade, v. 28, p. 25-47, 2015.

SALAZAR, Noel. Prefacio. In: ZUNINO SINGH, Dhan; GIUCCI, Guillermo; JIRÓN, Paola (orgs.). Términos clave para los estudios de movilidad en América Latina. Buenos Aires: Biblos, 2018, p. 9-11.

SALAZAR; Gabriela Cano; RAMÍREZ, Clemencia; CABRERA, Donna. Aproximación a la identificación de las corrientes migratorias procedentes de Asia y África en Colombia. In: Migrantes extracontinentales en América del Sur: Estudio de casos. Cuadernos Migratorios n. 5. Buenos Aires: Organización Internacional para las Migraciones (OIM), p. 22-83, 2013.

SANDOVAL GARCIA, Carlos. No más muros. Exclusión y migración forzada en Centroamérica. San José: Universidad de Costa Rica, 2015.

SINATTI, Giulia. Migraciones, transnacionalismo y locus de investigación: Multilocalidad y transición de "sitios" a "campos". In: SOLÉ, Carlota; PARELLA, Sònia; CAVALCANTI, Leonardo (orgs.). Nuevos retos del transnacionalismo en el estudio de las migraciones. Madrid: Ministerio de Trabajo e Inmigración, 2008, p. 95-112.

SJM-CR; RJM-CA. Migrantes extraregionales en tránsito por Centroamérica. Riesgos, desafíos y oportunidades para el acompañamiento. San José: Servicio Jesuita para Migrantes Costa Rica (SJM-CR) y Red Jesuita con Migrantes Centroamérica (RJM-CA), 2017. 
TAPIA LADINO, Marcela. Frontera, movilidad y circulación reciente de peruanos y bolivianos en el norte de Chile. Estudios Atacameños, n. 50, p. 195-213, 2015.

TEDESCO, João Carlos; KLEIDERMACHER, Gisele (orgs.). A imigração senegalesa no Brasil e na Argentina: múltiplos olhares. Porto Alegre: Est Edições, 2017.

TEDESCO, João Carlos. "Em nome de ...": religião, trabalho e mercado. Senegaleses em frigoríficos do centro-norte do Rio Grande do Sul. In: TEDESCO, João Carlos; KLEIDERMACHER, Gisele (orgs.). A imigração senegalesa no Brasil e na Argentina: múltiplos olhares. Porto Alegre: Est Edições, 2017, p. 311-338.

VOGT, Wendy A. Stuck in the Middle With You: The Intimate Labours of Mobility and Smuggling along Mexico's Migrant Route. Geopolitics, v. 21, n. 2, p. 366386, 2016.

VOGT, Wendy A. The arterial border: negotiating economies of risk and violence in Mexico's security regime. International Journal of Migration and Border Studies, v. 3, n. 2/3, p. 192-207, 2017.

WINTERS, Nanneke. Redrawing the Central American Migrant Caravan: How Other (African) Trajectories Cross Its Path. Border Crimonologies. 2018. Disponible en: <https://www.law.ox.ac.uk/research-subject-groups/centre-criminology/ centreborder-criminologies/blog/2018/11/redrawing-central>. Acceso en: 10.04.2019.

WINTERS, Nanneke; MORA IZAGUIRRE, Cynthia. Es cosa suya: entanglements of border externalization and African transit migration in northern Costa Rica. Comparative Migration Studies, v. 7, n. 27, 2019. Disponible: <https://doi. org/10.1186/s40878-019-0131-9>.

ZARAGOCIN CARVAJAL, Sofía; MOREANO VENEGAS, Melissa; ÁlVAREZ VELASCO, Soledad. Hacia una reapropiación de la geografía crítica en América Latina. Presentación del dossier. Íconos, Revista de Ciencias Sociales, n. 61, p. 11-32, 2018.

ZUBRZYCKI, Bernarda; AGNELLI, Silvina. "Allá en África, en cada barrio por lo menos hay un senegalés que sale de viaje". La migración senegalesa en Buenos Aires. Cuadernos de Antropología Social, n. 29, p. 135-152, 2009.

ZUBRZYCKI, Bernarda; AGNELLI, Silvina; OTTENHEIMER, Ana Cristina. La migración ghanesa en Buenos Aires. Una contribución a la caracterización de la presencia africana en Argentina. In: Congreso Nacional ALADAA Argentina, Buenos Aires, 20 a 21 de agosto de 2010.

ZUBRZYCKI, Bernarda. Recent African migration to South America: The case of Senegalese in Argentina. International Journal of Humanities and Social Sciences, v. 2, n. 22, p. 86-94, 2012.

ZUBRZYCKI, Bernarda; SÁNCHEZ ALVARADO, Lina Fernanda. Redes y proyectos migratorios de los Senegaleses en Argentina. Cadernos Ceru, v. 26, n. 1, 2016. 